\title{
Determination of serum and organ malondialdehyde (MDA) concentration, a lipid peroxidation index, in Trypanosoma brucei-infected rats
}

\author{
J. I. Eze • B. M. Anene • C. C. Chukwu
}

Published online: 4 September 2008

(C) Springer-Verlag London Limited 2008

Erratum to: Comp Clin Pathol

DOI 10.1007/s00580-008-0722-6

The abstract contained an erroneous passage of text:

Incorrect: Six rats each were sacrificed on days 0,14 and 28-postinfection. Five rats each were sacrificed on day 14 and day 28 post-infection (PI) from group B, and their organs were promptly collected and washed with normal saline and used for organ malondialdehyde (MDA) concentration.

Correct: Six rats each were sacrificed on days 0,14 and 28 post-infection, and their organs were promptly collected and washed with normal saline and used for organ malondialdehyde (MDA) concentration.

The online version of the original article can be found at http://dx.doi. org/10.1007/s00580-008-0722-6.

J. I. Eze $(\bowtie) \cdot$ B. M. Anene $\cdot$ C. C. Chukwu

Department of Veterinary Medicine, University of Nigeria,

Nsukka, Enugu State, Nigeria

e-mail: jamesifeeze@yahoo.com 